\title{
Navigation, localization and stabilization of formations of unmanned aerial and ground vehicles
}

\author{
Martin Saska, Tomáš Krajník, Vojtěch Vonásek, Petr Vaněk, and Libor Přeučil
}

\begin{abstract}
A leader-follower formation driving algorithm developed for control of heterogeneous groups of unmanned micro aerial and ground vehicles stabilized under a top-view relative localization is presented in this paper. The core of the proposed method lies in a novel avoidance function, in which the entire 3D formation is represented by a convex hull projected along a desired path to be followed by the group. Such a representation of the formation provides non-collision trajectories of the robots and respects requirements of the direct visibility between the team members in environment with static as well as dynamic obstacles, which is crucial for the top-view localization. The algorithm is suited for utilization of a simple yet stable visual based navigation of the group (referred to as GeNav), which together with the on-board relative localization enables deployment of large teams of micro-scale robots in environments without any available global localization system. We formulate a novel Model Predictive Control (MPC) based concept that enables to respond to the changing environment and that provides a robust solution with team members' failure tolerance included. The performance of the proposed method is verified by numerical and hardware experiments inspired by reconnaissance and surveillance missions.
\end{abstract}

\section{INTRODUCTION}

Recent progress in development of autonomous microscale vertical take-off and landing vehicles (so called Micro Aerial Vehicles - MAVs) allows us to consider their deployment in various applications, which are strictly dedicated to autonomous ground robots (UGVs) nowadays. In this paper, a scenario of multi-robot surveillance is investigated, where a formation of autonomous vehicles has to repeatedly drive through a workspace in a phalanx to cover a large operating space. MAVs can bring several advantages compared to UGVs in such a mission. For example, MAVs can reach locations inaccessible by UGVs and they may provide a top view survey of the scene, which provides an important overview for human supervisors. Nevertheless, MAVs are also handicapped by several reasons. They have low payload for sensors, they have lower operational range due to limited power source and they are difficult to control in workspaces constrained by obstacles (e.g. in abundant vegetation). These aspects make especially appealing to take advantage of both platforms and to employ a heterogeneous MAVs-UGVs team. Besides, the co-existence of ground and flying robots can provide efficient solutions of fundamental formation driving problems, as is a precise and reliable relative localization of team members closely cooperating together, which reduces probability of collisions within the robotic group.

The authors are with Department of Cybernetics, Faculty of Electrical Engineering, Czech Technical University in Prague, Technicka 2, 166 27 Prague 6, Czech Republic \{saska, vonasek, tkrajnik, preucil\}alabe.felk.cvut.cz
Usually, robots in reconnaissance and surveillance missions may not rely on pre-installed precise global localization infrastructures and commonly available systems (as GPS) lack required precision for control of compact formations. Besides, GPS lacks sufficient reliability mainly in urban and indoor environments. The proposed formation driving approach is adapted for an on-board visual relative localization, which uses simple light-weight cameras mounted on MAVs and identification patterns placed on UGVs and MAVs. With this top-view concept, one may better tackle the problem of loss of direct visibility that frequently occurs in visual relative localization of ground robots operating in a workspace with obstacles. The possibility of team members' relative localization from above increases precision and reliability of the localization and brings another perspective to see the scene by operators supervising the mission.

Beside the visual relative localization of individual robots, we propose to use a simple vision based technique also for the formation navigation in the environment. The presented formation driving method relies on a navigation approach called GeNav [1], which uses features detected in images gathered by a monocular camera carried by a leader of the formation. This very simple method enables to robustly navigate the group along a pre-learnt path consisting of a set of straight segments (a proof of stability of this method can be found in [1]).

The combination of the top-view relative localization and visual navigation provides a light-weight, low-cost, easyto-deploy and efficient solution, which may act as an enabling technique for extensive utilization of simple microscale robots. This paper is focussed on theoretical and implementing aspects of the formation driving mechanism suited for the real-world deployment of autonomous robots under the GeNav navigation and the top-view localization, while technical details on the visual relative localization are available in [2] and the GeNav navigation in [1].

The research endeavor in the formation driving community is aimed mainly at tasks of formation stabilization [3], [4], [5], [6], [7] and/or formation following a predefined path [8], [4], [9], [10], [11]. The algorithms are designed for UGV formations [3], [8], for unmanned vehicle (UAV) formations [4], [11], for MAV (mainly quadrotors) formations [9], [10], [6], [7] or even for heterogeneous MAV-UGV formations [5]. Most of the mentioned techniques are suited for utilization of robots under a precise external global localization system (for example approaches [7], [10] are verified with the VICON system), for UGV formations they often rely on a deadreckoning with its cumulative error [8] or they provide 
theoretical solutions verified only by simulations [3], [4], [6], [9], [11], where a known position of robots may be assumed. In our work, the necessity of utilization of on-board systems for robots' localization and navigation is inherently included in the essence of the formation driving approach. The system for stabilization of robots in the team is adapted for requirements of available robust localization and navigation techniques, which enables its utilization in real-world scenarios. In our method, we rely on the Model Predictive Control (MPC) to be able to involve the following constraints into the formation driving: Constraints imposed 1) by the inter vehicle relations (shape of the formation feasible for the top-view relative localization), 2) by vehicles (mobility constraints), 3) by obstacles (environment constraints) and 4) by the GeNav technique employed for the navigation of the entire group along straight line segments of the desired path.

The MPC approach is often used for stabilizing nonlinear systems with control constraints (see [12] and references reported therein for descriptions and a general survey of MPC methods). In the formation driving, researchers take advantage of MPC mainly to respond to changes in dynamic environment [11], [9], [10]. In [9] and [10] it was shown, that the computational power of microprocessors available onboard of unmanned helicopters enables to employ MPC techniques also for the formation control of such a high dynamic systems, similarly as it is proposed here. In our approach, we go beyond these papers in the following aspects. We apply the MPC method for the stabilization of the formation with included requirements of the top-view relative localization, which could be an enabling technique for deployment of heterogeneous MAVs-UGVs teams outside the laboratories (without any global localization as is e.g. the VICON in [10]). We present a novel obstacle avoidance function with a simple and effective representation of the $3 \mathrm{D}$ formation included. It provides a robust solution of the formation driving in environments with dynamic obstacles. Our formation driving method is designed for the purpose of simple yet stable visual navigation developed in [1], which is well suited for the reconnaissance and surveillance missions being our target applications. Beside the dynamic obstacles avoidance, the proposed method provides an intervehicle avoidance, which is crucial for failure tolerance of the system. All these behaviours and abilities are numerically and experimentally verified at the end of this paper.

\section{PROBlem STATEMENT AND PRELIMINARIES}

Development of method presented in this paper is motivated by reconnaissance applications, where a team of robots has to autonomously follow a desired path given by a supervising expert. During the movement along the path, the robots have to keep a formation suited for the mission requirements. The robots can form a searching phalanx (a line formation) to be able to search for victims or intruders in large areas or a compact fleet of vehicles can be used for transportation purposes. We assume that one robot of the group (UGV or MAV), called GeNav leader in this paper, is capable of navigation along such a path. We will employ a navigation system based on SURF features detection in an image provided by an on-board camera. The system was developed for navigation of a UGV robot [1] and later extended for an MAV [13]. This system (referred as GeNav) is suited for guidance of robots along path that consists of set of straight segments. Its precision $(\sim 20 \mathrm{~cm})$ and reliability enables a robust navigation of a single robot, but it is not sufficient for the coordination of the robots within a compact formation with small relative distances between robots.

Beside the GeNav leader, we assume a group of simple UGV followers without any on-board sensors for their localization and a group of MAV followers (quadrotors) equipped with a bottom camera and a system for visual relative localization [2]. This system provides information on the relative position between the camera of MAVs and center of an identification pattern. The identification patterns are carried by all UGVs and MAVs except the one flying in the highest altitude. The precision of the employed visual relative localization system $(\sim 1 \mathrm{~cm})$ is sufficient for the formation stabilization in the desired shape. We assume that the shape is designed in a way that all robots, except the MAV flying in the highest altitude, are in the field of view of at least one bottom camera mounted on an MAV.

Now, let us describe preliminaries important for description of the method, in which such a heterogeneous 3D formation of MAVs and UGVs has to follow the desired path, while requirements of the formation driving and the top-view relative localization are satisfied. It means that 1) the movement of the formation has to be smooth also in the unsmooth connections of path segments, where the GeNav leader is turning around on the spot, and 2) the direct visibility between the vehicles have to be kept during the formation deployment.

Let $\psi_{j}(t)=\left\{x_{j}(t), y_{j}(t), z_{j}(t), \varphi_{j}(t)\right\}$, where $j \in$ $\{G L, V L, 1, \ldots, n f\}$, denote configurations of the GeNav leader $G L$, a virtual leader $V L$, and $n f$ followers at time $t$. The GeNav leader is equipped with the on-board visual navigation to follow the pre-learnt path segments. $G L$ is positioned in front of the formation and it is used as a reference point fort the coordinate system using by the topview relative localization. Whereas, the virtual leader is a reference point for the proposed formation driving technique. $V L$ is initially placed in the same position and orientation as the GeNav leader. Using the trajectory following approach described in Section III-C, it keeps the same position as $G L$ except the deviation caused by obstacles that could brake the top view localization or to cause collisions. Significant deviation of $G L$ and $V L$ positions can be also seen in connections of line segments of the desired path. In these points, the path is not feasible for the formation of nonholonomic robots, which forces the virtual leader to temporarly leave the path to be able to follow a smooth trajectory feasible for the formation.

The Cartesian coordinates $x_{j}(t), y_{j}(t)$ and $z_{j}(t)$ define positions $\bar{p}_{j}(t)$ of all robots (leaders and followers) and $\varphi_{j}(t)$ denotes their heading. Both MAVs and UGVs (except the 
robot assigned as the GeNav leader) are denoted as followers in the presented approach. For the MAVs, the heading $\varphi_{j}(t)$ becomes directly the yaw (see Fig. 2 for the coordination system of MAVs). Roll together with pitch do not need to be included in the kinematic model employed in MPC, but they depend on the type of utilized MAVs as shown for a quadrotor in [14].

Let us assume that the environment contains a finite number $n_{0}$ of compact obstacles. The obstacles can be static (as part of a known map) or dynamic and unknown (detected during the formation movement by on-board sensors). These updates of the map are shared by all robots via a WiFi communication. A follower or even more followers of the formation can become dynamic obstacles if deviating from their desired positions as demonstrated in the failure tolerance simulation in section IV.

The kinematics for any robot $j$ in 3D is described by the simple nonholonomic kinematic model: $\dot{x}_{j}(t)=$ $v_{j}(t) \cos \varphi_{j}(t), \dot{y}_{j}(t)=v_{j}(t) \sin \varphi_{j}(t), \dot{z}_{j}(t)=w_{j}$ and $\dot{\varphi}_{j}(t)=K_{j}(t) v_{j}(t)$, where feed-forward velocity $v_{j}(t)$, curvature $K_{j}(t)$ and ascent velocity $w_{j}(t)$ represent control inputs denoted as $\bar{u}_{j}(t)=\left\{v_{j}(t), K_{j}(t), w_{j}(t)\right\}$. We assume that UGVs operate in a flat surface and that $z_{j}(\cdot)=0$ and $w_{j}(\cdot)=0$ for each of the UGVs. In case of MAVs, $v_{j}(\cdot)$, $K_{j}(\cdot)$ and $w_{j}(\cdot)$ values are inputs for the low level controller, as shown in [14].

Let us now describe a discretization of the kinematic model as it is used in the proposed formation driving with the model predictive trajectory following included. Let define a time interval $\left[t_{0}, t_{\text {end }}\right]$ consisting of a sequence of elements of increasing times $\left\{t_{0}, t_{1}, \ldots, t_{\text {end }-1}, t_{\text {end }}\right\}$, such that $t_{0}<$ $t_{1}<\ldots<t_{\text {end-1 }}<t_{\text {end }}$. We will refer to $t_{k}$ using its index $k$ in this paper. For the model predictive planning, the control inputs are held constant over each time interval $\left[t_{k}, t_{k+1}\right)$, where $k \in\{0, \ldots, e n d\}$. We will call the points at which the control inputs change as transition points. By integrating the kinematic model over these intervals, the following discretized model may be obtained:

$$
\begin{aligned}
& \text { if } K_{j}(k+1) \neq 0 \text { : } \\
& x_{j}(k+1)=x_{j}(k)+\frac{1}{K_{j}(k+1)}\left[-\sin \left(\varphi_{j}(k)\right)+\right. \\
& \left.\sin \left(\varphi_{j}(k)+K_{j}(k+1) v_{j}(k+1) \Delta t\right)\right], \\
& y_{j}(k+1)=y_{j}(k)-\frac{1}{K_{j}(k+1)}\left[-\cos \left(\varphi_{j}(k)\right)+\right. \\
& \left.\cos \left(\varphi_{j}(k)+K_{j}(k+1) v_{j}(k+1) \Delta t\right)\right], \\
& z_{j}(k+1)=z_{j}(k)+w_{j}(k+1) \Delta t \\
& \varphi_{j}(k+1)=\varphi_{j}(k)+K_{j}(k+1) v_{j}(k+1) \Delta t
\end{aligned}
$$

and if $K_{j}(k+1)=0$ :

$$
\begin{aligned}
x_{j}(k+1) & =x_{j}(k)+v_{j}(k+1) \cos \left(\varphi_{j}(k)\right) \Delta t, \\
y_{j}(k+1) & =y_{j}(k)+v_{j}(k+1) \sin \left(\varphi_{j}(k)\right) \Delta t, \\
z_{j}(k+1) & =z_{j}(k)+w_{j}(k+1) \Delta t \\
\varphi_{j}(k+1) & =\varphi_{j}(k)+K_{j}(k+1) v_{j}(k+1) \Delta t,
\end{aligned}
$$

where $x_{j}(k), y_{j}(k)$ and $z_{j}(k)$ are the rectangular coordinates and $\varphi_{j}(k)$ the heading angle at the transition point with index $k$. $\Delta t$ is a sampling time, which is uniform in the whole interval $\left[t_{0}, t_{\text {end }}\right]$. The control inputs $v_{j}(k+1), K_{j}(k+1)$ and $w_{j}(k+1)$ are constant between transition points with index $k$ and $k+1$.

As mentioned in the problem statement, we assume a heterogeneous 3D formation of a given shape, which satisfies the requirements given by the formation driving and the topview localization: 1) robots are in a safe relative distance; 2) each robot, except the MAV flying in the highest altitude, is observed by at least one MAV. In this paper, the shape of the entire formation is maintained with a leader-follower technique derived from the approach [15], which was designed for formations of UGVs working in a planar environment. For the heterogeneous MAV-UGV, we have extended the notation from [15] to 3D as visualized in Fig.1. Besides, we have extended the technique in [15], which is designed for following smooth splines (continuity of second-order is required), for utilization of paths consisting of straight line segments, which are required by the GeNav navigation.

In our method, both types of followers, MAVs and UGVs, follow the trajectory of the virtual leader in distances defined in $p, q, h$ curvilinear coordinate system. The position of each follower $i$ is uniquely determined by states $\psi_{V L}\left(t_{p_{i}}\right)$ in travelled distance $p_{i}$ from the actual position of the virtual leader along the virtual leader's trajectory, by offset distance $q_{i}$ from the trajectory in perpendicular direction and by elevation $h_{i}$ above the trajectory. $t_{p_{i}}$ is the time when the virtual leader was at the travelled distance $p_{i}$ behind its actual position. To get states of follower $i$ in rectangular coordinates, states of the virtual leader at time $t_{p_{i}}$, which is $\psi_{V L}\left(t_{p_{i}}\right)=\left\{x_{V L}\left(t_{p_{i}}\right), y_{V L}\left(t_{p_{i}}\right), z_{V L}\left(t_{p_{i}}\right), \varphi_{V L}\left(t_{p_{i}}\right)\right\}$, have to be shifted with vector $V\left(t_{p_{i}}\right)$ as follows:

$$
\psi_{i}(t)=\psi_{V L}\left(t_{p_{i}}\right)+V\left(t_{p_{i}}\right) .
$$

The vector $V\left(t_{p_{i}}\right)$ consists of four components: $V\left(t_{p_{i}}\right)=$ $\left(-q_{i} \sin \left(\varphi_{L}\left(t_{p_{i}}\right)\right), q_{i} \cos \left(\varphi_{L}\left(t_{p_{i}}\right)\right), h_{i}, 0\right)$.

\section{INTEGRATED TRAJECTORY PLANNING AND FORMATION STABILIZATION}

\section{A. Method overview}

The system designed for the stabilization of heterogeneous MAV-UGV formations is divided into three main blocks as you can see in the scheme depicted in Fig. 3. The first block, GeNav Leader, is responsible for navigation of the entire formation in the environment. It provides control inputs for the GeNav leader based on image features gained by an onboard camera. The GeNav method enables to navigate a robot or a group of robots along a pre-learnt path consisting of straight segments. The requirements on the piecewise straight desired path is important for stability of the method as analysed in [1].

From the formation stabilization perspective, an important output of the GeNav Leader module is a prediction of GeNav leader's states. For the prediction, it is assumed 

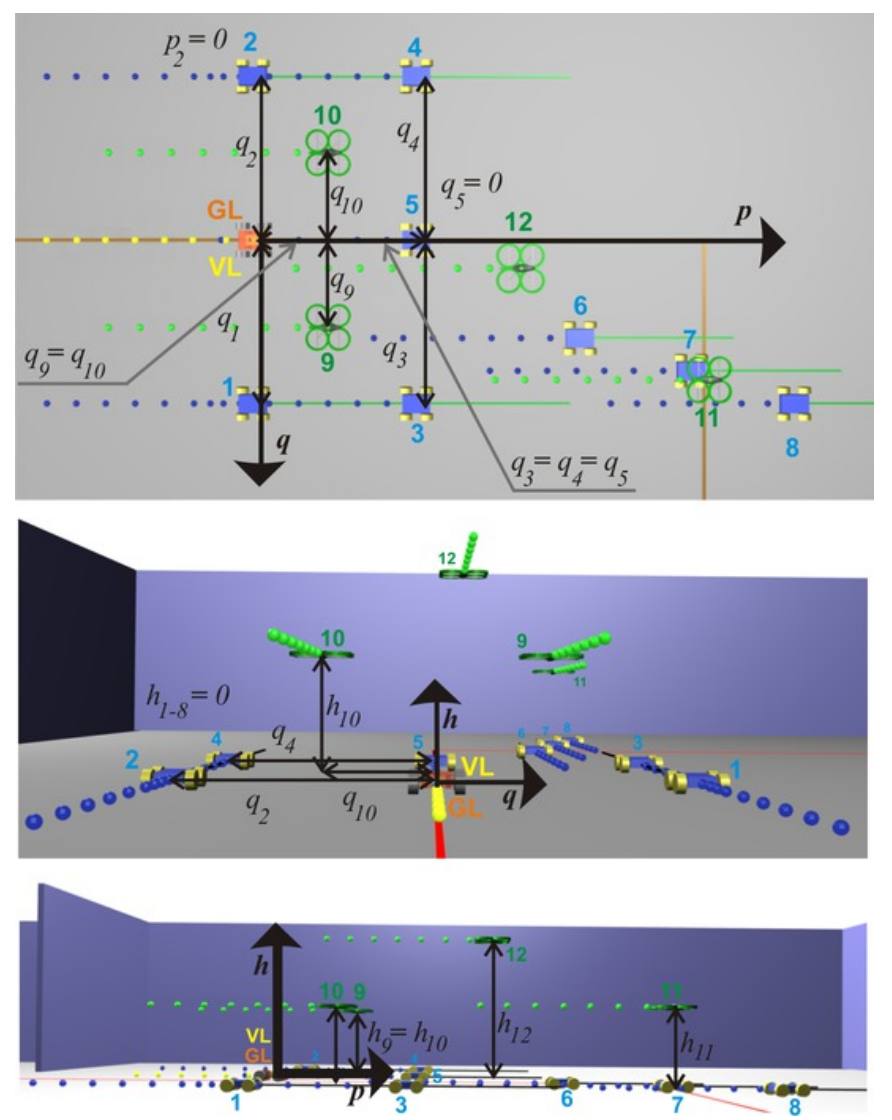

Fig. 1. The desired shape of the formation described in curvilinear coordinates.

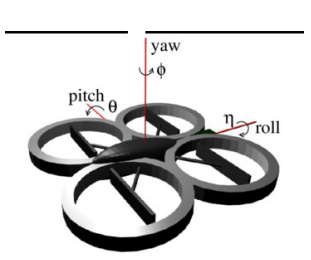

(a) MAV coordination system.

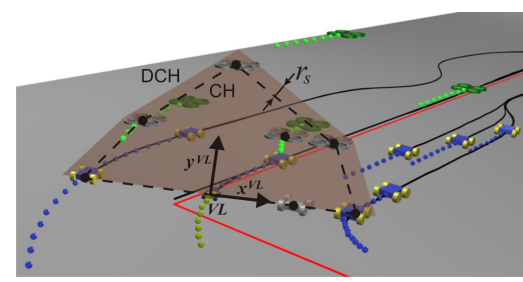

(b) Dilated convex hull. The shaded contours with black balls represent projections of followers into the plane of virtual leader.

Fig. 2. that the GeNav leader follows the desired path without any perturbation in both, the desired speed and the position on the path. The perturbations, which occur in real robotic systems, will be diminished by the presented receding horizon control technique. The predicted trajectory, which consists of $n$ states derived with constant sampling time $\Delta t$, acts as an input of the Virtual Leader block.

This part is important for avoidance of obstacles that could affect the relative localization within the group or that could collide with robots of the formation. Besides, it enables to follow the GeNav leader in connections of the line segments of the desired path. In the Virtual Leader part, the Trajectory Following block provides control inputs for the virtual leader, which is feasible for the entire formation and respects the requirements of the top-view relative localization

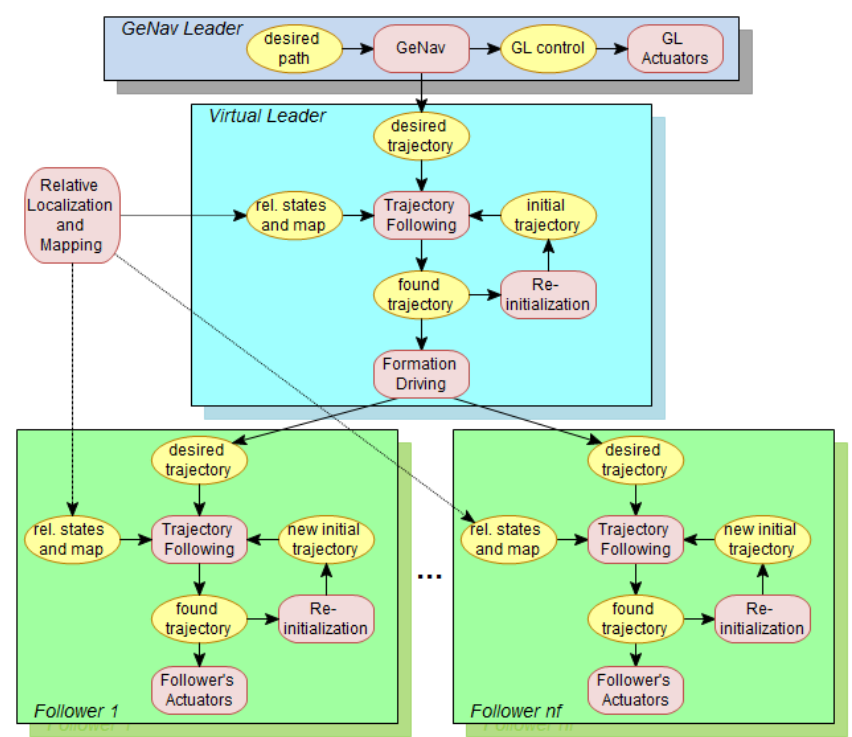

Fig. 3. Schema of the complete planning and control system.

via the model of the formation. In the straight segments of the desired path, the trajectory found by the Trajectory Following block follows the desired trajectory with minimal deviation and it is only employed to diminish possible perturbations. A significant difference between the desired and found trajectories occurs mainly due to appearing obstacles or near to line segment connections. Details on the trajectory following mechanism with emphasis on incorporation of the 3D heterogeneous formation stabilized under the top-view localization are presented in Section III-C.

The resulting trajectory obtained in the Trajectory Planning block is described by a sequence of configurations of the virtual leader $\psi_{L}(k)$, where $k \in\{1, \ldots, N\}$, and by constant control inputs applied in between the transition points. According the MPC concept, only a portion of the computed control actions is applied on the interval $\left\langle t_{0}, t_{0}+n \Delta t\right\rangle$, known as the receding step. This process is then repeated on the interval $\left\langle t_{0}+n \Delta t, t_{0}+N \Delta t+n \Delta t\right\rangle$ as the finite horizon moves by time steps $n \Delta t$, yielding a state feedback control scheme strategy. The unused part of the trajectory can be employed for re-initialization of the planning process in each planning step, since the plan of the formation between two consequent steps is usually changed only slightly.

In the proposed formation driving system, the trajectory obtained in the Trajectory Planning block is used as an input for the Formation Driving module, which transforms the plan to desired configurations of followers (using eq. (2)). The core of the third main block, which is multiplied for MAVs and UGVs followers, is also the Trajectory Following module. This part is responsible for avoiding of impending collisions with obstacles or other members of the team and it corrects deviations from the desired trajectory provided by the virtual leader. In real applications with dynamic obstacles and disturbances caused by the imprecise model of sensors and actuators, the desired trajectories provided by the Formation Driving cannot be directly applied for control 


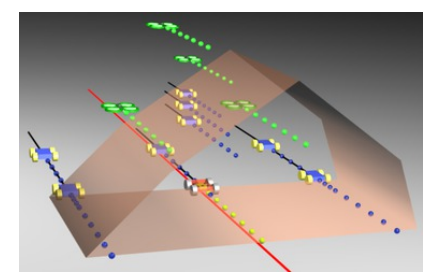

(a) Formation in a straight segment (b) Formation in a connection of line of the path.

segments.

Fig. 4. The dilated convex hull projected along the planned trajectory of the virtual leader.

of particular followers. They have to be adapted to ensure the stability of the group and non-collision movement. Similarly as in the leader's trajectory following, the unused part of the found trajectory can be employed for the initialization of the planning process.

\section{B. $3 D$ formation representation for the obstacle avoidance}

One of the main contribution of this paper is the ability of the system to ensure formation stabilization under the top-view visual relative localization in environments with dynamic obstacles. This requires to design an obstacle avoidance function included into the trajectory following methods, which are introduced in Fig. 3. The core of the avoidance function is a proper representation of the entire formation, which incorporates the requirement on the direct visibility between the robots into the formation stabilization process.

In our approach, the 3D formation is represented by a convex hull of positions of followers projected into a plane $\mathcal{P}_{V L}$, which is orthogonal to the trajectory of the virtual leader in its actual position (see Fig. 2(b)). The projection of the position of $i$-th follower into the plane $\mathcal{P}_{V L}$ can be simply obtained as $x_{i}^{V L}:=q_{i}$ and $y_{i}^{V L}:=h_{i} .\left\{x^{V L} ; y^{V L}\right\}$ is coordinate system in the plane $\mathcal{P}_{V L}$ as sketched in Fig. 2(b). The convex hull of the set of points $\left\{x_{i}^{V L} ; y_{i}^{V L}\right\}$, where $i \in\{1, \ldots, n f\}$, is an appropriate representation of the $3 \mathrm{D}$ formation under the top-view relative localization by two reasons: 1) Each follower $i$ intersects the plane $\mathcal{P}_{V L}$ at point $\left\{x_{i}^{V L} ; y_{i}^{V L}\right\}$ in future. 2) The convex hull of such a set of points denotes borders of the area, which should stay obstacle free. This ensures that the direct visibility between MAVs and UGVs, which is crucial for the presented top-view visual localization, is satisfied.

Moreover for the obstacle avoidance function presented in Section III-C, the convex hull $(\mathrm{CH})$ needs to be dilated by a detection boundary radius $r_{s}$ to keep obstacles in a desired distance from followers. Only obstacles that are closer to the convex hull than $r_{s}$ are considered in the avoidance function. In the trajectory following process applied for followers control, the DCH is reduced to a circle with radius equal to $r_{s}$ to represent a single robot.

\section{Trajectory planning and control for the virtual leader}

Let us now describe the trajectory following mechanism with obstacle avoidance function more in details. As mentioned above, the aim of the method is to find a control sequence that steers the virtual leader along the desired path followed by the GeNav leader and consequently to find control sequences that stabilize the followers behind the virtual leader in a desired relative positions. The intention of the method is to find the control sequences to keep the virtual leader as close as possible to the GeNav leader and followers as close as possible to their desired position behind the virtual leader, while satisfying the requirements given by the non-collision formation driving and the top-view relative localization. By applying this concept, the group is able to respond to changes in workspace, which can be dynamic or newly detected static obstacles, and to failures of a robot of the team.

To define the trajectory planning problem in a compact form, we need to gather states $\psi_{j}(k)$, where $k \in\{1, \ldots, N\}$ and $j \in\left\{V L, 1, \ldots, n_{r}\right\}$, into vector $\Psi_{j} \in \mathbb{R}^{4 N}$ and the control inputs $\bar{u}_{j}(k)$ into vector $\mathcal{U}_{j} \in \mathbb{R}^{3 N}$. All variables describing the trajectory of the virtual leader or a follower can be collected in an optimization vector: $\Omega_{j}=\left[\Psi_{j}, \mathcal{U}_{j}\right] \in \mathbb{R}^{7 N}$. Let us now transform the trajectory planning to minimization of a cost function $J_{j}\left(\Omega_{j}\right), j \in\left\{V L, 1, \ldots, n_{r}\right\}$ subject to sets of equality constraints $h_{j}(k)=0, \forall k \in\{0, \ldots, N-1\}$ and inequality constraints $g_{j}(k) \leq 0, \forall k \in\{1, \ldots, N\}$. The proposed cost function consists of four components:

$$
\begin{aligned}
& J_{j}\left(\Omega_{j}\right)=\sum_{k=1}^{N}\left\|\left(\bar{p}_{d, j}(k)-\bar{p}_{j}(k)\right)\right\|^{2} \\
& +\sum_{l=1}^{n_{o}}\left(\min \left\{0, \frac{d_{D C H}\left(\Omega_{j}, o_{l}\right)}{d_{D C H}\left(\Omega_{j}, o_{l}\right)-R_{D C H}}\right\}\right)^{2} \\
& +\frac{1}{N} \sum_{k=1}^{N}\left(v_{j}(k)-\bar{v}_{j}\right)^{2}+\left(K_{j}(k)-\bar{K}_{j}\right)^{2} \\
& +\sum_{f \in \bar{n}_{n}}\left(\min \left\{0, \frac{d_{j, f}\left(\Omega_{j}, \Omega_{f}^{\circ}\right)-r_{s, j}}{d_{j, f}\left(\Omega_{j}, \Omega_{f}^{\circ}\right)-r_{a, j}}\right\}\right)^{2} .
\end{aligned}
$$

The first part penalizes solutions with states deviated from the desired states $\bar{p}_{d, j}(k)$, where $k \in\{1, \ldots, N\}$. In the virtual leader's trajectory tracking, the desired states are obtained by the prediction of the movement of the GeNav leader. In the followers' trajectory planning, the desired states are derived from the result of the virtual leader's trajectory tracking using the formation driving concept for each of the followers.

The second term of $J_{j}\left(\Omega_{j}\right)$ contributes to the final cost when an obstacle is inside the projection of the dilated convex hull along the planned trajectory. As mentioned, the convex hull represents the formation in case of the virtual leader's trajectory planning or a single robot in case of the followers' trajectory planning. Examples of the projected convex hull are shown in Fig. 4. The value of second term of $J_{j}\left(\Omega_{j}\right)$ will be increasing as the obstacle is approaching to the centre of the convex hull. The constant $R_{D C H}$ is equal to half of the maximal width of the dilated complex hull measured in the $x^{L}$ coordinate $\left(R_{D C H}=r_{s}\right.$ in the followers' trajectory planning). The function $d_{D C H}\left(\Omega_{j}, o_{l}\right)$ 
provides distance from the dilated convex hull to obstacle $o_{l}$ again in the direction of $x^{L}$ coordinate. The function value is negative if the obstacle is outside the dilated convex hull and positive if the obstacle is in the hull. The direction of the gradient of such defined avoidance function is to the side of the hull in the $x^{L}$ coordinate. This is important since the formation, which is "fixe" by UGVs to the ground, cannot avoid obstacles by change of its altitude.

The third term is important for reducing of undesirable oscillations in movement of robots and it eliminates needless aggressive manoeuvres. This term penalises high variance of control inputs. During the optimization process, solutions with control inputs deviating from their mean values, $\bar{v}_{j}=$ $\frac{1}{N} \sum_{k=1}^{N} v_{j}(k)$ and $\bar{K}_{j}=\frac{1}{N} \sum_{k=1}^{N} K_{j}(k)$, are penalized, which results into smooth trajectories.

Finally, the last part of cost function $J_{j}\left(\Omega_{j}\right)$ is crucial for the failure tolerance of the system. This term is a sum of avoidance functions in which the other members of the team are considered also as dynamic obstacles. This part has to protect the robots in case of an unexpected behaviour of a defective neighbour. Function $d_{j, f}\left(\Omega_{j}, \Omega_{f}^{\circ}\right)$ provides minimal distance between the planned trajectory of $j-t h$ follower $\Omega_{j}$ and the recent plan of $f$-the robot $\Omega_{f}^{\circ}$. The $(\cdot)^{\circ}$ symbol denotes of the last results of the optimization process for the particular robot. The minimal distance is provided for all $f \in$ $\bar{n}_{n}$, where $\bar{n}_{n}=\left\{1, \ldots, j-1, j+1, \ldots, n_{r}\right\}$. The detection radius $r_{s, j}$ is usually smaller than the basic detection radius $r_{s}$ used for the dilation of the convex hull, because the follower should not try to avoid a close neighbour if both are at the desired position. Besides, the detection radius, we need to define a circular avoidance boundary with radius $r_{a, j}$, where $r_{s, j}>r_{a, j}$. While, single robots should not respond to other followers detected outside the region with radius $r_{s, j}$, distance between the robots and their neighbours less than $r_{a, j}$ is considered as inadmissible (it could cause a collision).

The equality constraints $h(k)$ represent the kinematic model (1) for all $k \in\{0, \ldots, N-1\}$ with initial conditions given by the actual state of the leader. This ensures that the obtained trajectory stays feasible with respect to kinematics of utilized robots. It means that these constraints are satisfied if $\psi_{j}(k+1)$ is obtained by substituting the vectors $\psi_{j}(k)$ and $\bar{u}_{j}(k+1)$ into the eq. (1) for all $k \in\{0, \ldots, N-1\}$.

The sets of inequality constraints $g(k)$ characterize bounds on control inputs $\bar{u}_{j}(k)$ for all $k \in\{1, \ldots, N\}$. For all followers, the control inputs are limited by vehicle mechanical capabilities (i.e., chassis and engine) as $v_{\min , i} \leq$ $v_{i}(k) \leq v_{\max , i},\left|K_{i}(k)\right| \leq K_{\max , i}$ and for MAVs also $w_{\min , j} \leq w_{j}(k) \leq w_{\max , j}$. These values may differ for each of the followers. For the virtual leader, these limits have to be extended, since the constraints of the entire formation need to be included. The trajectory of the virtual leader must be feasible for all followers in their desired positions. For the virtual leader, the admissible control set can be determined using the leader-follower approach as $\max _{i=1, \ldots, n_{r}}\left(\frac{-K_{\max , i}}{1-q_{i} K_{\max , i}}\right) \leq K_{V L}(k) \leq$
TABLE I

CURVILINEAR COORDINATES OF FOLLOWERS WITHIN THE FORMATION USED IN THE EXPERIMENT PRESENTED IN FIG. 5-6.

\begin{tabular}{c||l|l|l|l|l|l|l|l|l|l|l|l}
$i$ & 1 & 2 & 3 & 4 & 5 & 6 & 7 & 8 & 9 & 10 & 11 & 12 \\
\hline$p_{i}$ & 0 & 0 & 1 & 1 & 1 & 2 & 2.7 & 3.4 & .5 & .5 & 2.7 & 1.6 \\
$q_{i}$ & 1 & -1 & 1 & -1 & 0 & .6 & .8 & 1 & .5 & -.5 & .8 & .2 \\
$h_{i}$ & 0 & 0 & 0 & 0 & 0 & 0 & 0 & 0 & .5 & .5 & .5 & 1
\end{tabular}

$\min _{i=1, \ldots, n_{r}}\left(\frac{K_{\max , i}}{1+q_{i} K_{\max , i}}\right)$ and $\max _{i=1, \ldots, n_{r}}\left(\frac{v_{\min , i}}{1+q_{i} K_{L}(t)}\right)$ $\leq v_{V L}(k) \leq \min _{i=1, \ldots, n_{r}}\left(\frac{v_{\max , i}}{1+q_{i} K_{L}(t)}\right)$. These restrictions must be applied to respect different values of curvature and speed of robots in different positions within the guided formation. Intuitively, e.g. the robot following the inner track during a turning movement goes slower but with a bigger curvature than the robot further from the center of the turning.

\section{EXPERIMENTAL RESULTS}

The aim of the simulation presented in Fig. 5- 6 is to show performance of the presented method in a complex scenario with static and dynamic obstacles. It enables to clearly present main abilities of this approach in representative situations inspired by a real world mission. In the experiment, the formation driving technique is employed in a surveillance application, in which a heterogeneous team of MAVs and UGVs has to periodically move through three rooms connected by a corridor. The objective of the mission is to follow a given path and to keep a desired shape of the formation. The formation can be autonomously temporarily shrunk in narrow passages (e.g. in doorways) or due to dynamic obstacles forcing followers to perform avoidance manoeuvres.

The initial position of the group is depicted in Fig. 5(a). As described in Fig. 1, the formation consists of the GeNav leader (the orange robot denoted by $G L$ ), the virtual leader (the yellow robot denoted by $V L$ ), $8 \mathrm{UGV}$ followers and 4 MAV followers. Three of the MAVs are positioned in a lower altitude to be able to relatively localize the ground robots. The fourth MAV is flying above them to provide relative positions of the lower MAVs. Besides, the desired relative positions of MAVs in the formation are determined in such a way that they are not mutually influenced by air flow effects. The followers' coordinates relative to the virtual leader are in Table I.

Results presented in this section have been obtained using the proposed algorithm with parameters: $n=2, N=8$ and $\Delta t=0.25 \mathrm{~s}$. We have employed the SQP method [16] for solving the optimization problems used in the virtual leader trajectory tracking and for the stabilization and obstacle avoidance of followers. This solver provided the best performance from the tested available algorithms. Nevertheless, one can use any optimization method, which is able to solve the optimization problems defined in this paper.

In the first detailed snapshot of the simulation (Fig. 5(b)), the outer followers of the formation temporarily deviate from 


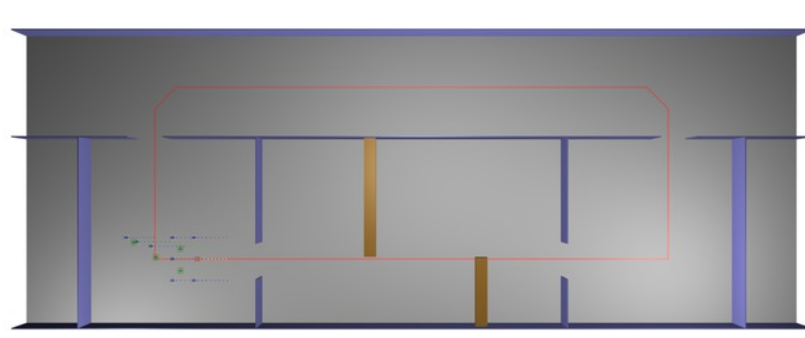

(a) Initial position. $\mathbf{t}=\mathbf{1 s}$
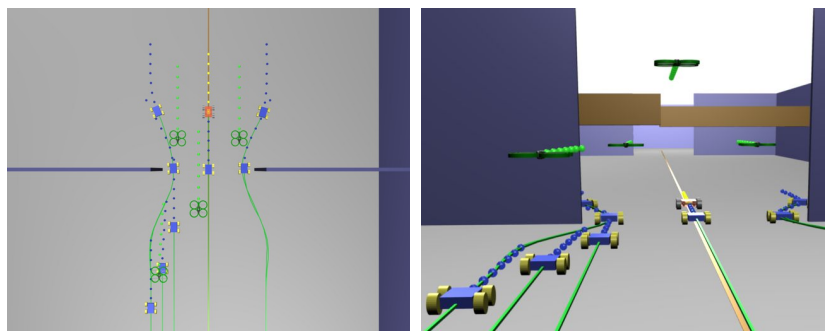

(c) Temporarily formation shrinking. $\mathbf{t}=\mathbf{2 3 s}$

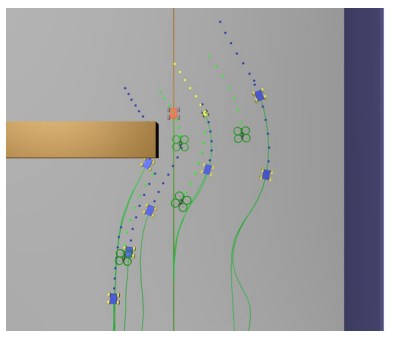

(e) 1 st overhead obstacle avoidance. $\mathbf{t}=\mathbf{5 5} \mathrm{s}$

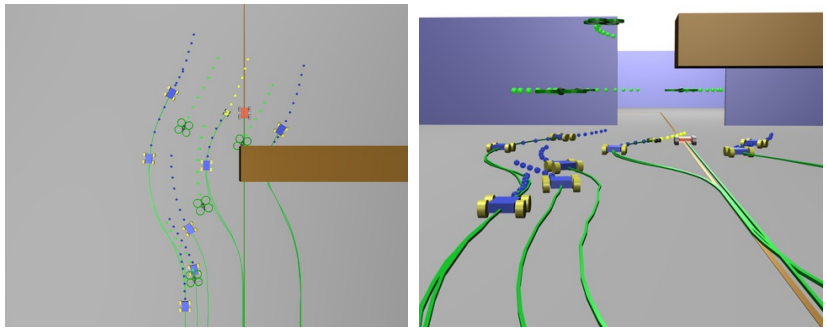

(g) 2nd overhead obstacle avoidance. $\mathbf{t}=\mathbf{9 1 s}$
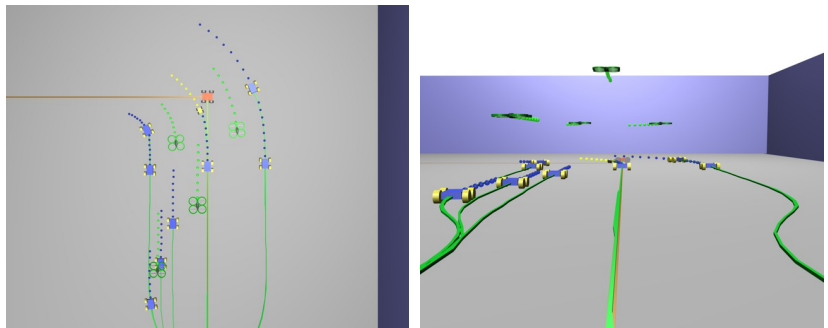

(i) GeNav leader finished the turning on spot. $\mathbf{t}=\mathbf{1 7 1 \mathrm { s }}$
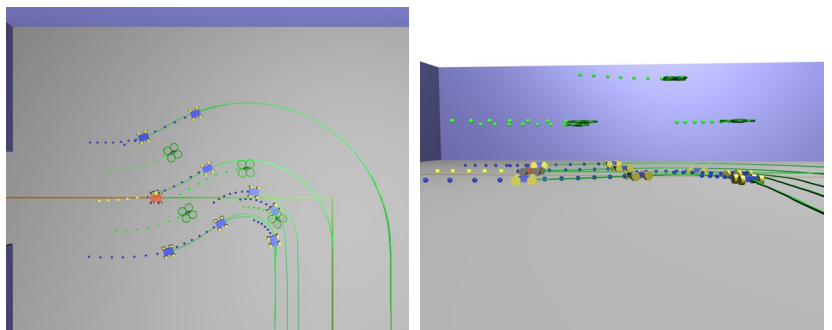

(k) The formation going back on the desired path. $\mathbf{t}=\mathbf{1 9 2} \mathrm{s}$
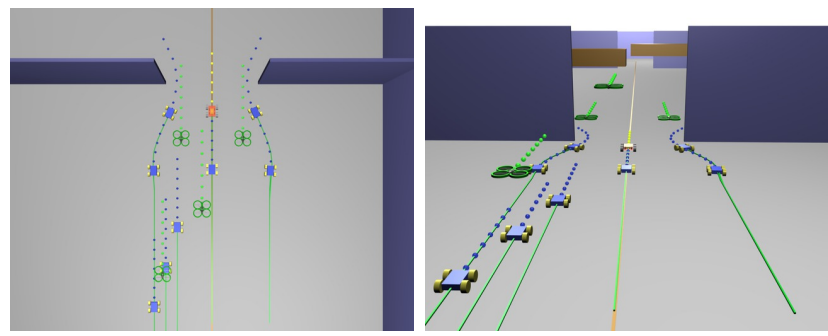

(b) Response to a narrow passage. $\mathbf{t}=\mathbf{1 6} \mathbf{s}$

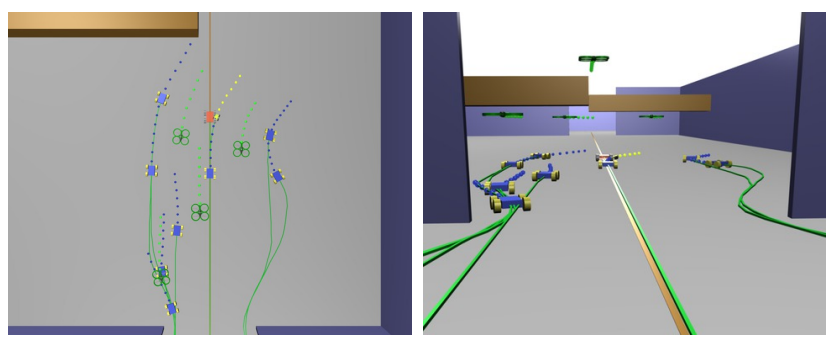

(d) Response to the 2 nd overhead obstacle. $\mathbf{t}=\mathbf{4 2} \mathrm{s}$

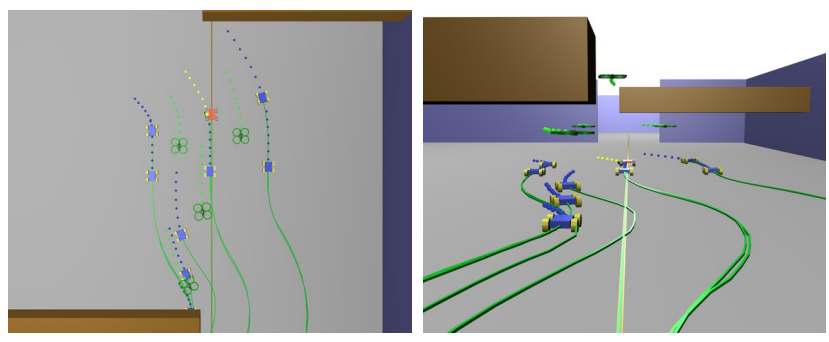

(f) Response to the 2 nd overhead obstacle. $\mathbf{t}=\mathbf{7 4 s}$

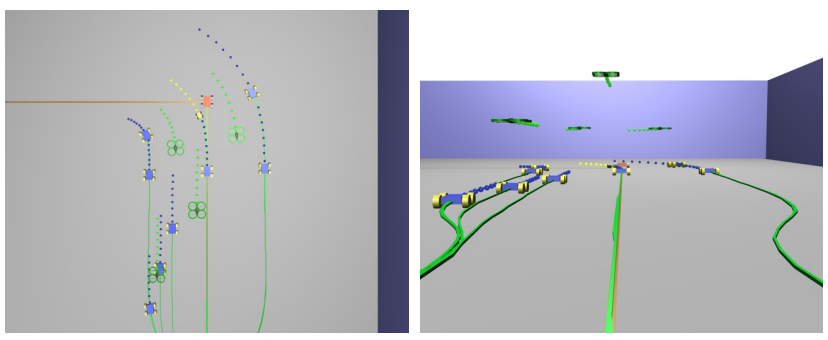

(h) 1 st connection of line segments reached. $\mathbf{t}=\mathbf{1 4 0 s}$

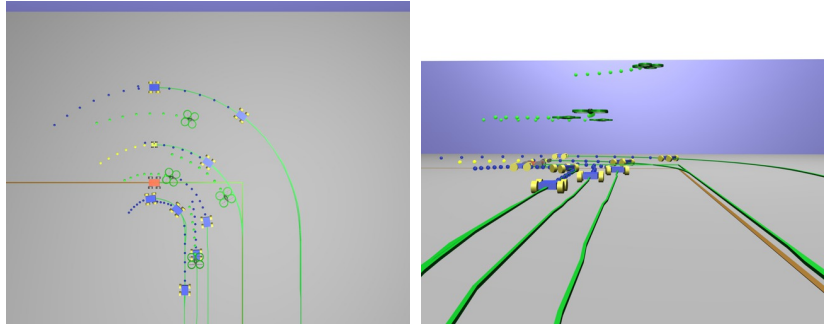

(j) The formation smoothly passing along the lines connection. $\mathbf{t}=\mathbf{1 8 2} \mathrm{s}$

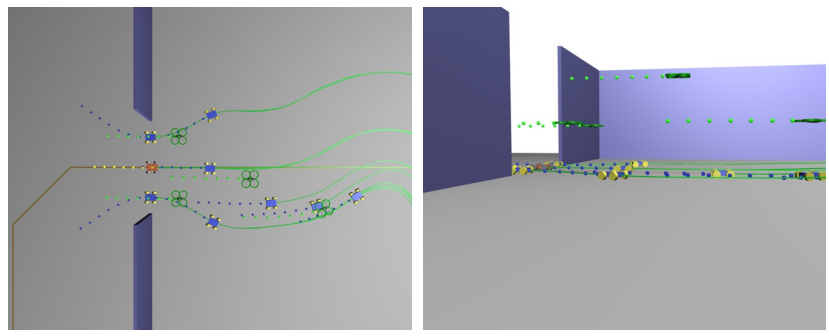

(l) Temporarily formation shrinking in the door into the corridor. $\mathbf{t = 2 0 7} \mathbf{s}$

Fig. 5. The first part of snapshots of the formation movement simulation. 

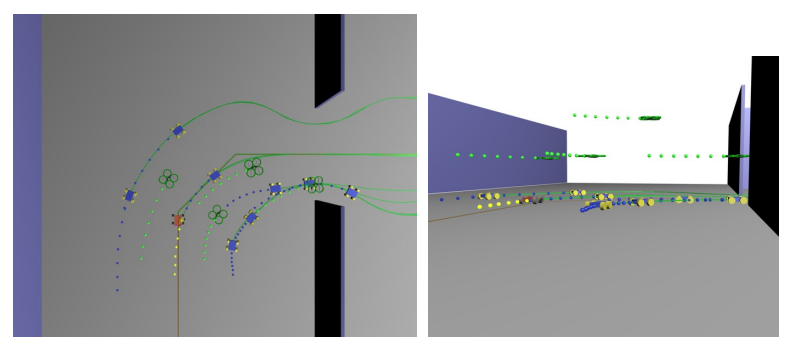

(a) Movement along connections of line segments. $\mathbf{t}=\mathbf{2 5 7} \mathrm{s}$

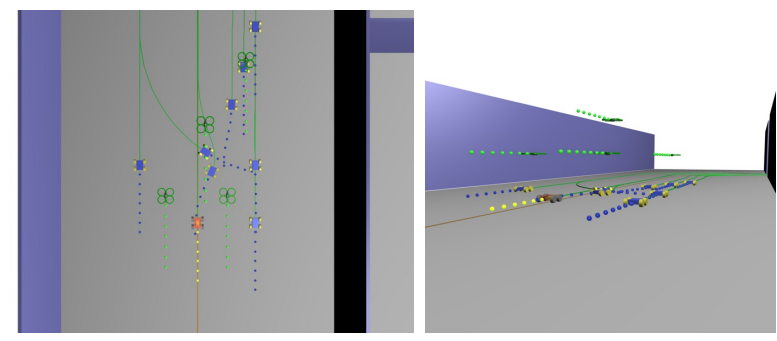

(c) Follower 5 avoiding the broken robot. $\mathbf{t}=\mathbf{2 9 8 s}$

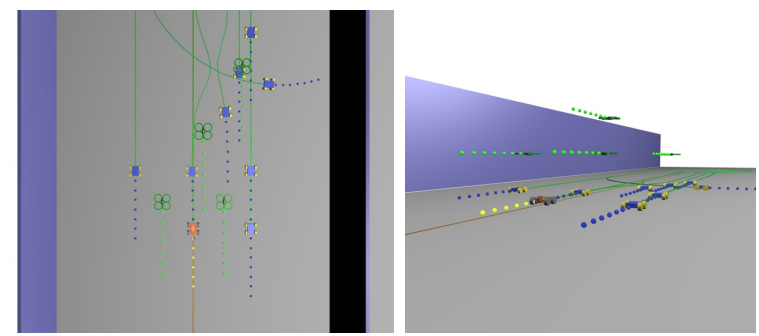

(e) All followers successfully avoided the broken robot. $\mathbf{t}=\mathbf{3 0 8 s}$

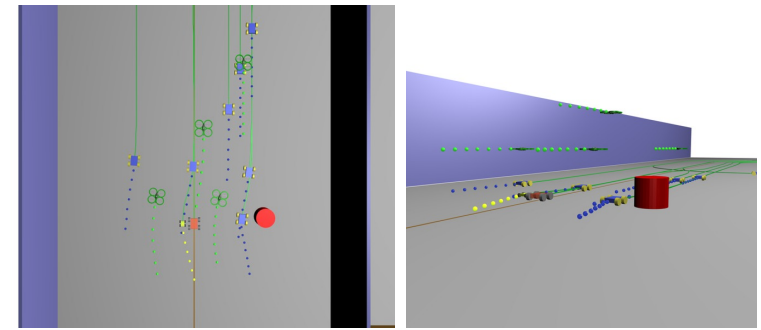

(g) Obstacle avoidance by the virtual leader's re-planning. $\mathbf{t}=\mathbf{3 2 3 s}$

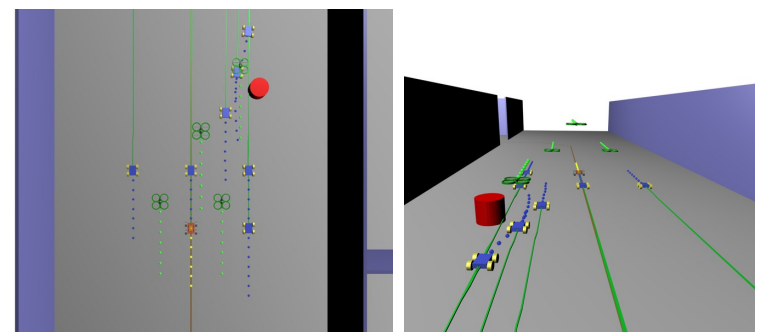

(i) 2nd (dynamic) obstacle detected by followers. $\mathbf{t}=\mathbf{3 6 2 \mathrm { s }}$
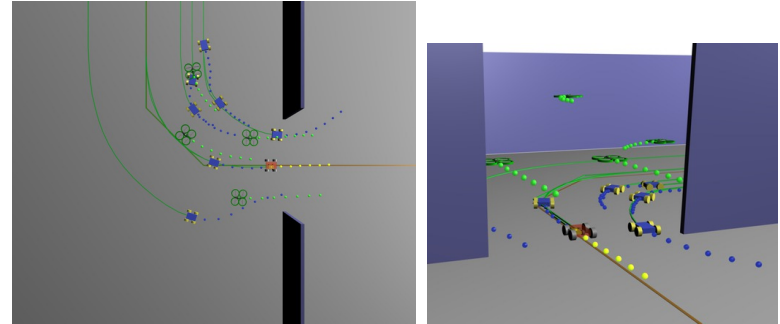

(k) Formation is shrinking to pass through the door. $\mathbf{t}=\mathbf{4 3 6} \mathrm{s}$

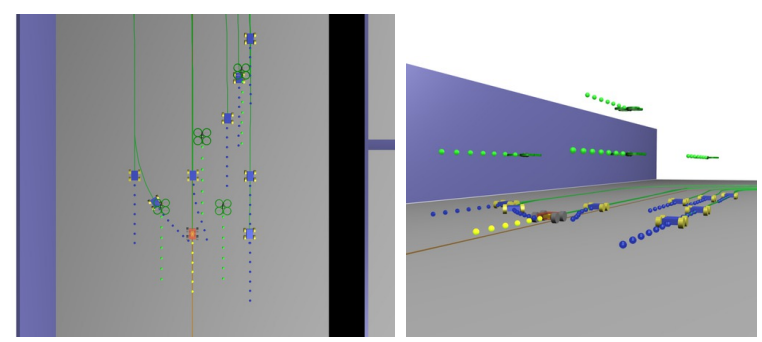

(b) Failure of follower 2 deviating from its desired position. $\mathbf{t}=\mathbf{2 8 9} \mathrm{s}$

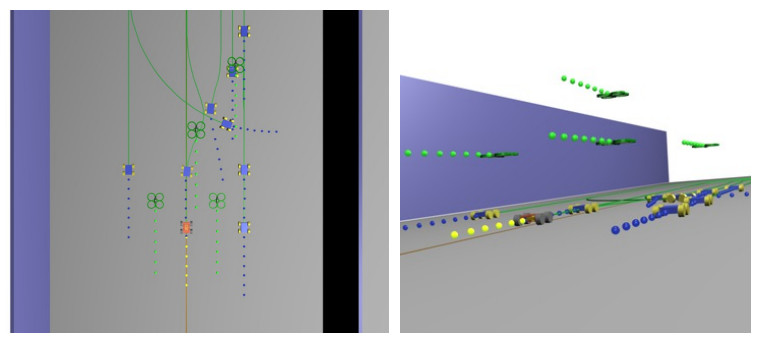

(d) Follower 6 avoiding the broken robot. $\mathbf{t = 3 0 3 s}$

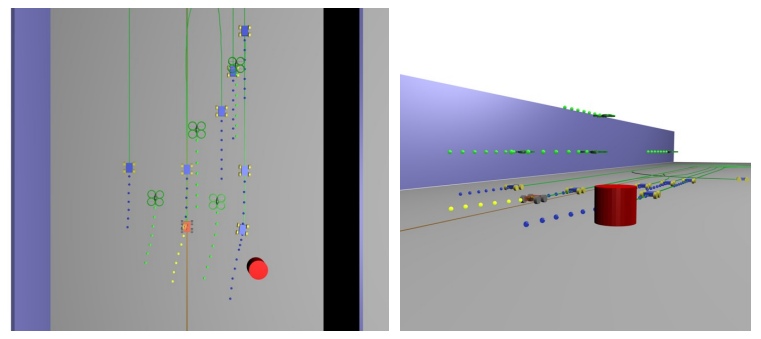

(f) 1 st unknown obstacle detected. $\mathbf{t}=\mathbf{3 1 8 s}$

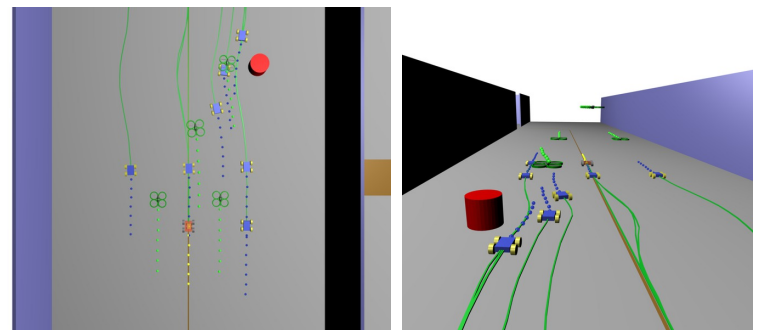

(h) All robots follow the leader to avoid the obstacle. $\mathbf{t}=\mathbf{3 3 9} \mathrm{s}$

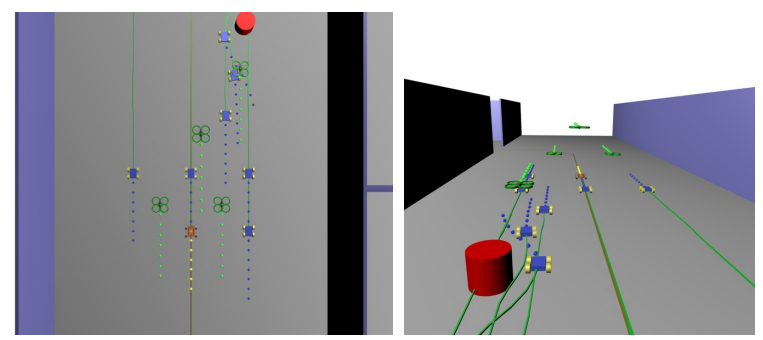

(j) Temporarily change of the formation to avoid the obst. $\mathbf{t}=\mathbf{3 6 9} \mathrm{s}$

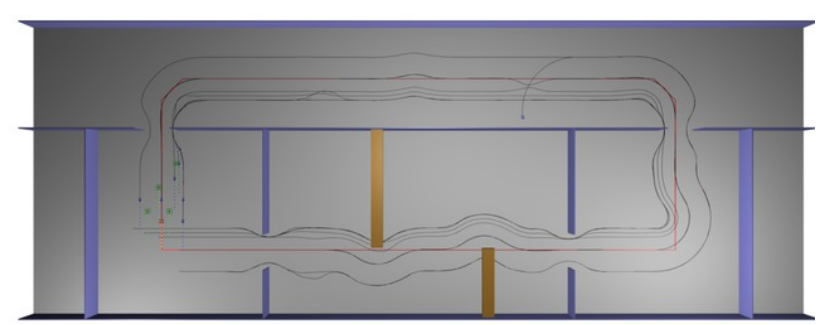

(1) Complete trajectories passed by the formation. $\mathbf{t}=\mathbf{4 7 7} \mathrm{s}$

Fig. 6. The second part of snapshots of the formation movement simulation. 
their desired positions to pass through the narrow passage towards the second room. In Fig. 5(d), the original shape of the formation is restored and the group starts avoiding the overhead obstacle. The obstacle is sufficiently high to be passed under by all robots except the MAV flying in the highest altitude. The GeNav leader can be navigated without any influence of the obstacle, but the rest of the formation has to move away from the desired path to keep the constraints given by the relative localization. In Fig. 5(d), one can see the deviation of position of the virtual leader from position of the GeNav leader. This enables to avoid the obstacle in a way that the obstacle is always situated outside the dilated convex hull of the formation. In Fig. 5(f), the formation returns back on the desired path, but it is again forced to avoid the second overhead obstacle. In Fig. 5(h), the GeNav leader is approaching into the first connections of line segments of the path. The virtual leader and the followers are waiting for the GeNav leader, which has to turn on the spot. They are already deviated from the path to be able to smoothly continue without any complicated manoeuvring. Once the turning of the GeNav leader is finished (Fig. 5(i)), the complete formation continues back on the desired path. Fig. 6(a) presents movement of the formation along connections of path segments that make a less sharp angle. The deviation of the formation from the desired path is significantly lower than in the previous case of the 90 degree edge of the path. In Fig. 6(b)-6(e), a failure of one of the followers (its steering was blocked) has been simulated to show failure tolerance and robustness of the system. In Fig. 6(c), one can see a successful avoidance manoeuvre of follower 5 and a changed plan of follower 6 as a response to prediction of the collision (see the last part of eq. (3) for details on the applied avoidance function). In Fig. 6(f), an unknown obstacle is detected by the formation. The obstacle is avoided, using the virtual leader's obstacle avoidance function, at the price of temporarily leaving of the desired path (see Fig. 6(g)6(h)). The second obstacle is detected by the followers in Fig. 6(i). This dynamic obstacle cannot be avoided by the virtual leader's re-planning, since it was detected too late. Therefore, the avoidance function included in the follower's trajectory following method is utilized here. The shape of the formation is temporarily change to keep the obstacle outside the dilated convex hull.

Cost-function values of the virtual leader's and the 1st follower's trajectory planning during the movement presented in Fig. 5- 6 are depicted in Fig. 7. The peaks in the course of the leader's cost values correspond with the places of connections of line segments forming the desired path that has to be followed. In these connections, the virtual leader is forced to deviate from the path to be able to pass the sharp edges of the path smoothly. Also the first unknown obstacle is contributing into the virtual leader's cost values. The temporal increase of cost values of the trajectory tracking of follower 1, which was chosen as an interesting example, is caused by the proximity of the obstacles, which force the robots to leave the desired position in the formation. The deviation from the desired state is penalized by the first term

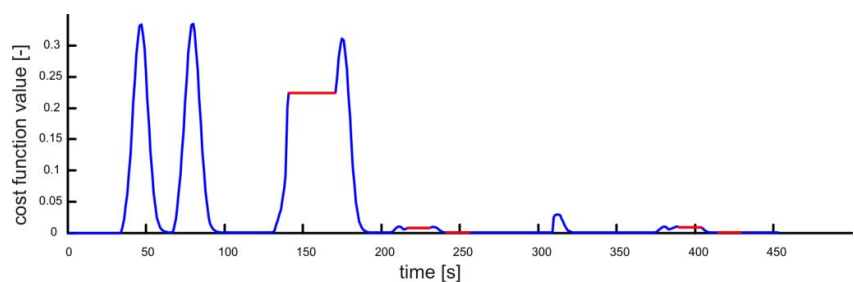

(a) The virtual leader.

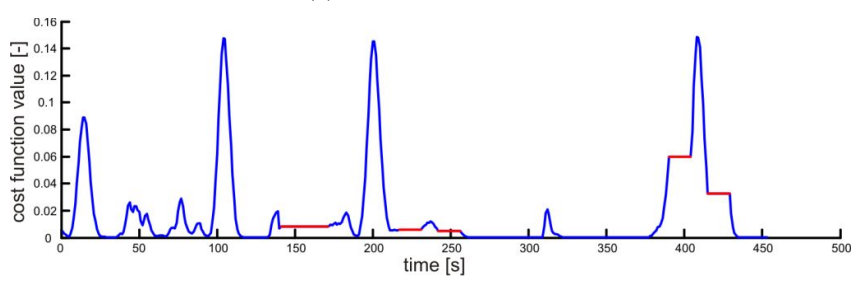

(b) The follower 1 .

Fig. 7. Values of the cost function employed in the trajectory planning method during the movement presented in Fig. 5- 6.

in the eq. (3). The red lines in the courses of the values denote parts, in which the GeNav leader is turning to be able to follow the next path segment and the rest of the formation is waiting in static positions.

The experiment in Fig. 8(a) demonstrates the ability of the obstacle avoidance by temporary shrinking of the formation and it verifies the formation movement in a connection of path segments (Fig. 8(b)). In the experiment, the Pioneer 3-AT robotic platform is employed as the GeNav leader and two MMP5 platforms and the Ar.Drone MAV act as followers. In Fig. 8 and in a video record of the experiment [17], beside the pictures of the formation movement, images used for the GeNav visual navigation and for the top-view relative localization are shown.

\section{CONCLUSION}

A novel formation driving algorithm suited for the stabilization of heterogeneous UGV-MAV teams under the topview visual relative localization and for navigation of the group based on the GeNav technique was presented in this paper. It was shown that utilization of such simple onboard vision based systems enables to deploy teams of closely cooperating unmanned ground and aerial vehicles outside laboratories; in environment without any pre-installed infrastructure for robots' localization. The abilities of the developed method to avoid static as well as dynamic obstacles and to avoid inter-vehicles collisions in case of a follower's failure were verified in simulations and hardware experiments.

\section{ACKNOWLEDGEMENTS}

This work was supported by GAČR under M. Saska's postdoc grant no. P10312/P756, by MŠMT under project Kontakt II no. LH11053, by the EU FP7 under the project REPLICATOR No. ICT-216240 (co-financed by MŠMT under the grant no. 7E08006) and under the project 

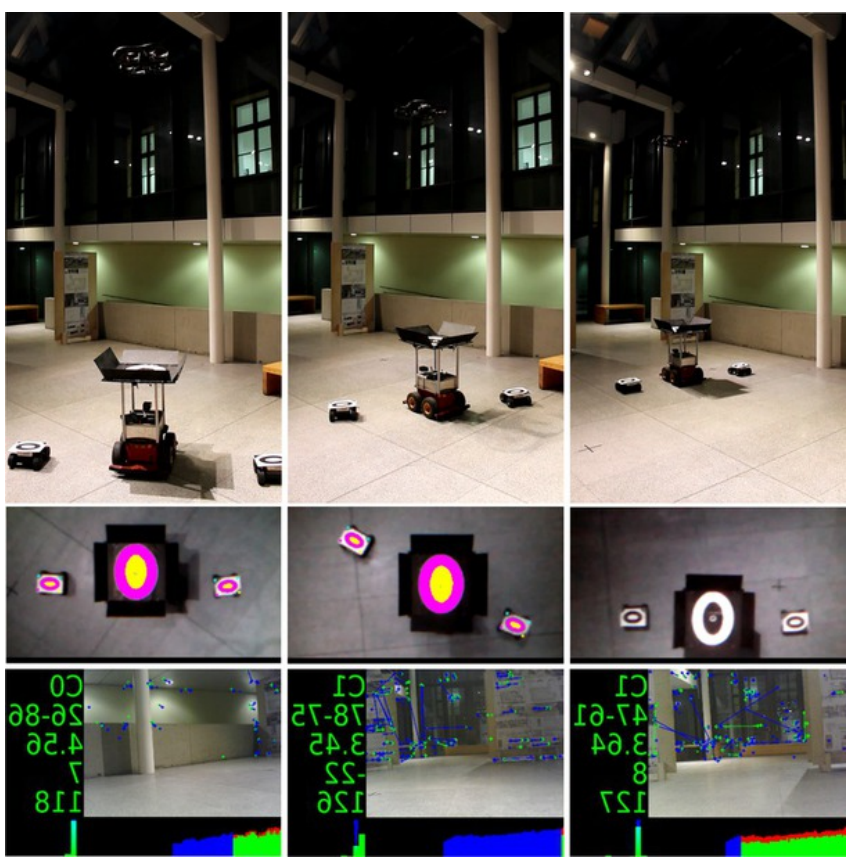

(a) Formation going through a connection of straight segments of the path.
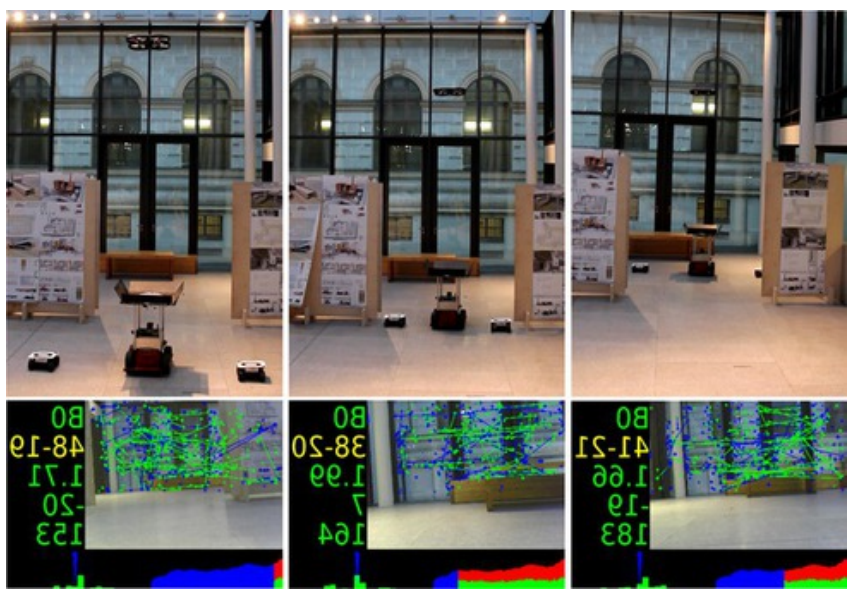

(b) Formation is temporarily shrinking to get through the narrow passage.

Fig. 8. Formation driving using the GeNav algorithm for the navigation and the top-view relative localization for the stabilization.

SYMBRION No. ICT-216342 (co-financed by MŠMT under the grant no. 7E11017), and by CTU under grant SGS12/145/OHK3/2T/13.

\section{REFERENCES}

[1] T. Krajník, J. Faigl, M. Vonásek, V. Kulich, K. Košnar, and L. Přeučil, "Simple yet stable bearing-only navigation," J. Field Robot., 2010.

[2] M. Saska, T. Krajník, and L. Přeučil, "Cooperative micro uav-ugv autonomous indoor surveillance," in IEEE SSD, 2012.

[3] Y. Liu and Y. Jia, "An iterative learning approach to formation control of multi-agent systems," Systems \& Control Letters, vol. 61, no. 1, pp. $148-154,2012$.

[4] T. S. No, Y. Kim, M.-J. Tahk, and G.-E. Jeon, "Cascade-type guidance law design for multiple-uav formation keeping," Aerospace Science and Technology, vol. 15, no. 6, pp. 431 - 439, 2011.

[5] H. Tanner and D. Christodoulakis, "Decentralized cooperative control of heterogeneous vehicle groups," Robotics and Autonomous Systems, vol. 55, no. 11, pp. $811-823,2007$.
[6] A. Abdessameud and A. Tayebi, "Formation control of vtol unmanned aerial vehicles with communication delays," Automatica, vol. 47, no. 11 , pp. $2383-2394,2011$.

[7] M. Turpin, N. Michael, and V. Kumar, "Trajectory design and control for aggressive formation flight with quadrotors," Auton. Robots, vol. 33, no. 1-2, pp. 143-156, 2012.

[8] J. Ghommam, H. Mehrjerdi, M. Saad, and F. Mnif, "Formation path following control of unicycle-type mobile robots," Robotics and Autonomous Systems, vol. 58, no. 5, pp. 727 - 736, 2010.

[9] M. Saffarian and F. Fahimi, "Non-iterative nonlinear model predictive approach applied to the control of helicopters group formation," Robotics and Autonomous Systems, vol. 57, no. 67, pp. 749 - 757, 2009.

[10] C. Liu, W.-H. Chen, and J. Andrews, "Piecewise constant model predictive control for autonomous helicopters," Robotics and Autonomous Systems, vol. 59, no. 78, pp. 571 - 579, 2011

[11] Z. Chao, S.-L. Zhou, L. Ming, and W.-G. Zhang, "Uav formation flight based on nonlinear model predictive control," Mathematical Problems in Engineering, vol. 2012, no. 1, pp. 1-16, 2012.

[12] M. Alamir, Stabilization of Nonlinear Systems Using RecedingHorizon Control Schemes, ser. Lecture Notes in Control and Information Sciences. Berlin / Heidelberg: Springer, 2006, vol. 339.

[13] T. Krajník, M. Nitsche, S. Pedre, L. Přeučil, and M. Mejail, "A Simple Visual Navigation System for an UAV," in International MultiConference on Systems, Signals and Devices. Piscataway: IEEE, 2012, p. 34

[14] T. Krajník, V. Vonásek, D. Fišer, and J. Faigl, “AR-Drone as a Platform for Robotic Research and Education," in Research and Education in Robotics: EUROBOT 2011. Heidelberg: Springer, 2011.

[15] T. D. Barfoot and C. M. Clark, "Motion planning for formations of mobile robots," Robotics and Autonomous Systems, vol. 46, pp. 65-78, February 2004.

[16] J. Nocedal and S. J. Wright, Numerical Optimization. Springer, 2006.

[17] Movie, "Movie of hw experiment and simulation presented in this paper [online]. [cit. 2013-2-22]. http://imr.felk.cvut.cz/formation/," 2013. 